\title{
La conception de l'aménagement des cours d'eau dans le cadre de la sauvegarde de l'environnement : L'exemple de la Fecht (Haut-Rhin)
}

\author{
PAR
}

\author{
F. Clément \\ Ingénieur en Chế du Gênie Rural, des Eaux et des Forêts \\ Directeur départemental de l'Agriculture \\ du Hant-Rhin
}

La plaine d'Alsace est drainée sur toute sa longueur, du sud au nord, par l'Ill qui se jette dans le Rhin à Strasbourg et reçoit, tout au long de son parcours, un certain nombre d'affiuents importants, pratiquement tous issus du massif vosgien.

La Fecht, située au nord-ouest du département du Haut-Rhin, a un bassin versant d'environ $510 \mathrm{~km}^{2}$. Elle prend sa source à $1137 \mathrm{~m}$, au pied du Breisfirst et du Lauchenkopf et se jette, après un parcours de $50 \mathrm{~km}$ environ, dans l'Ill à Illhaeusern dont l'altitude n'est que de $175 \mathrm{~m}$.

La pente de ce cours d'eau varie d'amont en aval de 24 à $0,8 \%$ et conduit à distinguer successivement :

- un cours supérieur caractérisé, à l'aval de Munster, par une érosion généralisée et un important débit solide de charriage;

- un cours moyen où alternent tronçons d'érosion et de dépôt;

- un cours inférieur qui constitue le type même du tronçon d'atterrissement, avec une large section transversale et une tendance aux méandres.

De la même façon, on peut distinguer, du point de vue géologique :

- le massif vosgien, composé essentiellement de granit, plus rarement de gneiss et de grès;

- les collines sous-vosgiennes, à l'est de la grande faille séparant le fossé rhénan du massif hercynien, et comportant des terrains variés qui constituent des restes de la couverture secondaire qui enveloppait le massif avant les mouvements tectoniques tertiaires;

- la plaine, où les alluvions récentes se limitent à une étroite bande le long des cours d'eau principaux, le reste étant constitué par un manteau de loess plus ou moins décalcifié par endroits en lehm.
La végétation, quant à elle, est essentiellement caractérisée par une très forte proportion de forêts $(54 \%)$, essentiellement dans la partie vosgienne du bassin versant.

Ia pluviométrie est très variable d'une extrémité à l'autre de la vallée, variant en année moyenne de $2 \mathrm{~m}$ sur la crête à $500 \mathrm{~mm}$ dans la plaine.

L'exploitation des stations limnigraphiques mises en place à Walbach, Bennwihr et Freland permet de chiffrer aux valeurs suivantes les débits caractéristiques du cours d'eau à Walbach:

- débit caractéristique d'étiage: $800 \mathrm{l} / \mathrm{s}$ (pouvant se réduire à $80 \mathrm{l} / \mathrm{s}$ );

- module : $5 \mathrm{~m}^{3} / \mathrm{s}$

- moyenne des crues annuelles: $70 \mathrm{~m}^{3} / \mathrm{s}$;

- maximum de la crue centenaire $: 180 \mathrm{~m}^{3} / \mathrm{s}$.

Les variations saisonnières de débit se manifestent par un étiage principal en période estivale, auquel s'ajoute éventuellement un étiage hivernal pendant les périodes de gel.

Les hautes eaux résultent le plus souvent d'une fusion rapide de la couverture nivale, plus ou moins accélérée par des précipitations relativement chaudes (coups de foehn).

La Fecht, à l'aval de Munster, est entretenue par deux syndicats fluviaux, associations syndicales forcées de droit local constituées par ordonnance du 6 novembre 1890 modifiée en 1918. Dirigés par un comité-directeur désigné par le Préfet, ces syndicats assurent la maîtrise d'ouvrage de tous les travaux d'aménagement du cours d'eau et perçoivent des cotisations sur les personnes morales et physiques en profitant (propriétaires riverains auxquels d'ailleurs se sont substituées volontairement les communes, syndicats d'irrigation, industriels, propriétaires de voies de communication protégées).

C'est à la suite des très fortes crues de janvier 1955, qui causèrent d'importants dommages aux ouvrages publics et privés, s'élevant à plusieurs centaines de millions d'anciens 


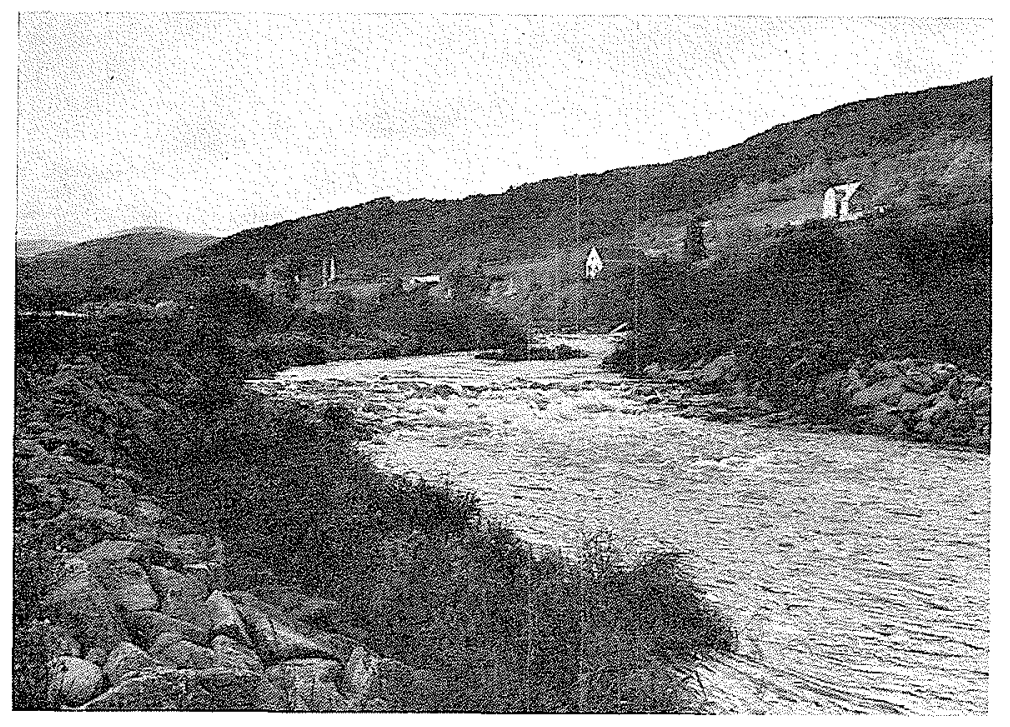

protection plus sommaire, voire une absence de protection de la rive convexe.

Il importait néanmoins, dans un premier temps, de consolider ou de remplacer certains ouvrages en ruine et de protéger les secteurs les plus menacés. Ces interventions locales, qui furent entreprises dès 1956, s`intégraient néanmoins dans le programme général qui, depuis bientôt vingt ans, est progressivement mis en œuvre en fonction des ressources des syndicats fluviaux (actuellement de $67061 \mathrm{~F}$ et $50714 \mathrm{~F}$ par an pour chacun des syndicats).

Grâce à l'intervention financière du Conseil Général du Haut-Rhin, qui subventionne à $40 \%$ de tels travaux, et aux subventions du Ministère de l'Agriculture, l'autofinancement résiduel, de l'ordre de 10 à $20 \%$, couvert par emprunts, a permis de réaliser 4 millions de francs de travaux sur ce cours d'eau.

\section{1/ La Fecht. Protection de rives et rampe en enrochements entre Gunsbach et Munster.}

francs, qu'a été mis sur pied un programme général d'aménagement du cours de la Fecht à l'aval de Munster.

L'important charriage constaté lors des crues sur les tronçons d'érosion situés le plus à l'amont et l'engravement constaté plus à l'aval nous ont amené à mettre en œuvre les deux principes suivants :

- stabilisation du profil en long, par création d'un certain nombre de biefs ayant pour pente la pente d'équilibre résultant des matériaux constitutifs du lit et séparés par des seuils fixes;

- stabilisation des rives dont les matériaux alluvionnaires résistent mal à l'érosion, en maintenant, dans toute la mesure du possible, un tracé en plan à base de courbes et de contre-courbes de rayon supérieur à cinq fois la largeur du cours d'eau, permettant, le plus souvent, unc

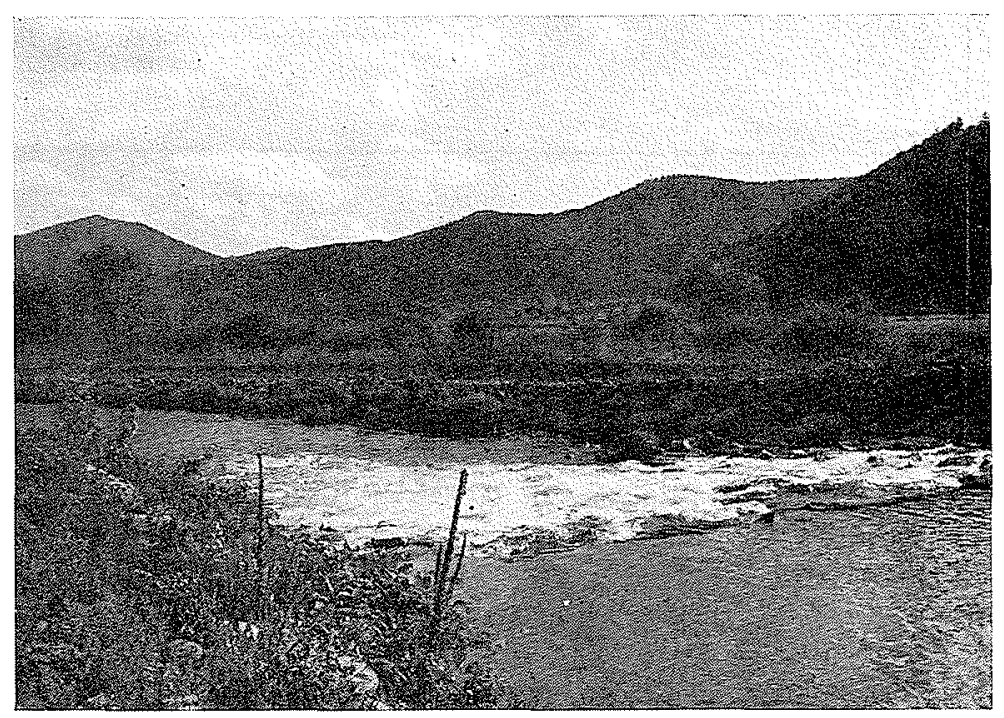

2/ Rampe d'enrochements sur la Fecht. Observer l'effet de centrage en résultant.

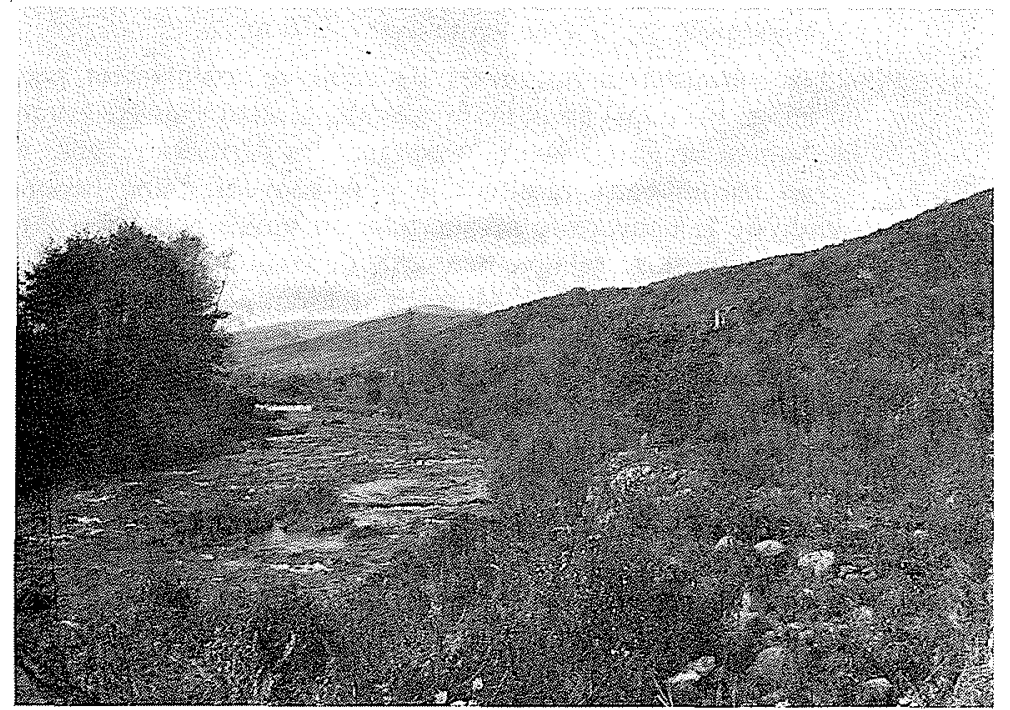

3/ Aspect général des rives de la Fecht quelques années après mise en place de boutures cie saule entre les enrochements de rives.
A l'exception des seuils dont la hauteur est supérieure à $1,50 \mathrm{~m}$, conçus comme des ouvrages à profil Craeger avec cuvette de dissipation le plus généralement pavée, la quasi totalité des ouvrages exécutés sont en enrochements.

Cette technique est inspirée des méthodes mises au point dans les Alpes autrichiennes il y a une vingtaine d'années et consiste à créer des rampes dont la pente est généralement de $10 \%$ et dont la hauteur est limitée au $1 / 20^{\circ}$ de la largeur du cours d'eau. Leur forme en plan est une courbe dont le rayon est égal à $5 / 4$ de la largeur du cours d'eau, afin de centrer le flot de crue à l'aval de l'ouvrage. Une flèche de l'ordre de $1 / 80^{\circ}$ de la longueur de l'ouvrage est prévue dans le même but pour centrer les débits d'étiage.

Ces rampes sont constituées de gros blocs de granit d'au moins une tonne, juxtaposés, mis en œuvre 
comme un gros hérisson particulièrement rugueux et absorbant l'énergie cinétique de l'eau. La rangée amont est enrobée de béton et la partie aval est retenue par une ou deux rangées de rails battus à $4 \mathrm{~m}$ de profondeur et espacés de 60 à $80 \mathrm{~cm}$

Un tel système présente les avantages suivants :

- bonne intégration au paysage;

- facilité de construction par suite de l'absence de fondations:

- possibilité de travailler sous l'eau sans épuisement;

- déformation possible sans ruine de l'ouvrage;

- bonne aération de l'eau favorisant l'autoépuration;

- remontée facile des poissons.

Cette solution nous paraît mériter d'être généralisée, dans la mesure où on dispose, à proximité, de matériaux valables, c'est-à-dire ayant un poids spécifique élevé, et non gélifs.

Ces mêmes matériaux sont utilisés, mais en blocs plus petits de 100 à $300 \mathrm{~kg}$, pour la protection des rives. L'emploi du béton est, en effet, limité au maximum et les perrés maçonnés sont réservés, soit à des ouvrages plus complexes comme des seuils comportant une prise d'eau, soit à la traversée de certaines agglomérations. Quant aux gabions, ils ont été rapidement abandonnés, étant donné d'une part la difficulté de trouver la main-d'œuvre nécessaire pour les remplir et, d'autre part, l'attaque par les eaux agressives de leur grillage, même galvanisé.

Ces enrochements, autrefois mis en place à la main en écailles de poisson de l'aval vers l'amont, sont maintenant manipulés à la pelle mécanique, à l'exception des petits blocs interstitiels placés entre les gros.

Leur fondation est constituée également par un massif d'enrochements mis en place dans une souille trapézoïdale.

Afin, par ailleurs, d'améliorer l'esthétique générale du cours d'eau, sont mises en place, de préférence en automne, des boutures de saules (salix purpurea) disposées, soit en lit sous les enrochements, soit le plus souvent implantées plus ou moins verticalement entre les blocs.

En quelques années est ainsi constitué un rideau de verdure cachant les enrochements et qu'il convient de recéper régulièrement tous les cinq ans.

Un tel dispositif reconstitue rapidement un paysage naturel et laisse parfois douter des interventions faites précédemment.

N'est-ce pas, en définitive, la plus grande satisfaction, à la fois pour les ingénieurs responsables de l'aménagement que pour les promeneurs particulièrement nombreux le long de nos rivières?

\section{Discussion}

M. le Président ouvre la discussion en ces termes:

Je remercie $M$. Clément de cette belle présentation. Qu'il s'agisse d'un grand aménagement ou d'un aménagement de rivière plus modeste, la qualité de la réalisation n'est pas liée à l'importance quantilative de la voie et les efforts qui ont été faits dans le cas présent sont significatifs de la possibilité de concilier ce qui, en apparence, est inconciliable, c'est-à-dire l'intervention de l'homme et le retour à une nature pratiquement identique à l'origine.

N'avez-vous pas des problèmes d'entretien pour empêcher une végétation trop luxuriante de s'établir sur les enrochements, ce qui gênerait l'écoulement des eaux?

Nous disposons, répond M. ClÉMENT, de deux gardes-rivière qui assurent l'entretien des plantations: c'est un eflectif minimum pour qu'un tel système soit valable.

Sur une question de M. le Président, M. Clément précise que les enrochements utilisés proviennent d'une carrière assez proche de la vallée de Munster; l'intérêt économique de cette technique est liś à la proximité des carrières sans laquelle le coût du transport devient sans commune mesure avec le prix du matériau lui-même.

Il est vrai, observe M. le Président, que la crise de l'énergie et l'augmentation du coût des produits - notamment du bitume et du ciment - peuvent conduire les ingénieurs à repenser le mode d'exécution des défenses de berges. L'utilisation des enrochements naturels qui, autrefois, était considérée comme dispendieuse, est peut-être devenue plus économique ane des procédés jusqu'ici jugés moins chers. Encore faut-il au'on n'ait pas trop de difficultés du point de vue de l'environnement pour ouvrir des carrières! mais là encore il est possible de procéder à des aménagements ou plutôt à des réaménagements des carrières pour les replacer harmonieusement dans leur cadre naturel.

Sur la demande de M. Jarror, M. Clément apporte les renseignements ci-après, sur le montant des travaux et leur mode de financement:
La Fecht est une rivière non navigable ni flottable et non un cours d'eau domanial.

Le financement des travaux d'hydraulique décrits est basé sur une subvention du Ministere de l'Agriculture de $50 \%$ et sur une subvention départementale de $40 \%$, le complément étant fourni par un prêt du Crédit Agricole à un taux relativement intéressant. Les ressources annuelles des deux syndicats fluviaux sont de l'ordre de $100000 \mathrm{~F}$ au total.

Si l'on veut mener à terme un tel programme général d'aménagement de cours d'eau, il est nécessaire de le subventionner très largement. Le Conseil Général l’a heureusement compris. Le réseau hydraulique peut être comparé, à mon sens, à un réseau routier; j'entends par-là qu'on peut comparer à la voirje nationale les cours d'eau navigables domaniaux; par contre, on peut assimiler à une voirie départementale les cours d'eau relativement importants comme le sont les principa!ix affuents de l'll1. Personnellement, je trouve très normal qu'une assemblée départementa'e subventionne au taux relativement important de $40 \%$ les principaux cours d'eau d'un département. C'est cetie politique, actuellement mise en ouvre, qui permet de lancer chaque année des tranches de travaux de l'ordre de $200000,300000 \mathrm{~F}$ et, surtout, de les poursuivre d'une année sur l'autre dans le cadre de ce programme général d'aménagement.

Il y a, remarque M. JARrot, une catégorie de cours d'eau encore plus défavorisée que ceux dont a parlé M. Clément; c'est celle des cours d'eau rayés de la nomenclature et restés dans le domaine public; on ne dispose généralement plus daucun moyen pour fairo des aménagements sur ces cours d'eau; or, certains d'entre eux, déclassés et rayés de la nomenclature depuis cinquante ans, commenment à poser des problèmes assez sérieux de divagation, d'érosion de berges, de destruction des barrages au fil de l'eau (donc de suppression des plans d'eau), d'envahissement des lits par la végétation (ce qui a une conséquence sur les crues). Il y a là un problème.

C'est en effet un problème inquiétant, estime $M$. le Président, car l'effort de l'Etat pour les voies navigables doit se concentrer sur 
les principales artères, les voies les moins «circulées» étant déclassées du réseau des voies navigables. Reste alors le problème de leur entretien; c'est également le problème des autres voies domaniales qui n'ont jamais été navigables mais continuent à relever de la responsabilité de l'Etat. La solution réside dans la conjonction de l'action de l'Etat, qui doit assurer ses responsabilités, et celle des collectivités locales. L'analyse de la philosophie du département du Haut-Rhin, que M. Clément nous a exposée, me paraît tout à fait valab'e quant à l'analogie entre le réseau routier et le réseau des voies navigables. C'est dans ce sens, par une action concertée de l'Etat et des collectivités locales, que doit être résolu le problème, car ces rivières constituent des biens collectifs et il est normal que les collectivités publiaues apportent leur participation à leur entretien. C'est un problème de bonne utilisation des ressources publiques.

Le débat revient sur le plan technique avec la question suivante de M. Carlier:

Comple tenu de la densité importante du réseau hydrométrique existant dans le département du Haut-Rhin, je suppose que la détermination du débit, pris en compte pour le recalibrage des cours d'eau, résulte d'une analyse statistique des débits observés.

Je souhaiterais savoir quelle est la durée de retour de la crue ainsi adoptée pour le recalibrage des cours d'eau et si le choix de cette durée de retour résulte d'une étude d'optimisation économique.

Une étude économique portant sur les inondations de la plaine de l'lll a été faite, répond M. Clément. Elle démontre que la lutte contre les inondations n'est en général pas rentable, sauf dans certains secteurs urbanisables à brève échéance. Les travaux d'aménagement des cours d'eau dans le Haut-Rhin n'ont donc pas pour but de supprimer les inondations éventuellement constatées, mais de stabiliser le lit; d'une manière générale, les sections sont calibrécs pour évacuer la crue décennale.

M. Hauville (DDE, Indre-et-Loire) formule ainsi son opinion: Le recalibrage des cours d'cau de faible importance est souvent relativement onéreux vis-à-vis des dommages causés par les inondations. Il semb!e souhaitable d'adopter, nour la détermination des sections à donner au lit de ces rivières, un débit égal à celui de la crue annuelle en zones boisées ou cultivées et de la crue décennale en traversée des lieux habités.

M. CrémEN est tout à fait d'accord avec M. Hauville Iorsqu'il s'agit de créer on d'agrandir le chenal d'évacuation. En l'occurence, pour les cours d'eau vosgiens, il ne s'agit que de stabiliser la section et le profil en long par une protection de berges. On peut alors, sans inconvénient, prendre en compte la crue décennale car la section existante correspond sensiblement à ce débit.

M. le Président clôt la discussion en remerciant M. Clément de son exposé ct des photographies très convaincantes qui l'accompagnaient. Il donne ensuite la parole à M. BLANIC pour l'exposé de la communication qu'il a rédigée en collaboration avec $M$. VERDET.

\section{Abstract \\ The problem of reconciling river development with environmental protection The example of the river Fecht in upper Alsace}

Author describes how development work was put into effect on the river Fecht, a tributary of the river 111 in the Haut-Rhin "Département" of France.

To counteract erosion caused by the river's substantial bet load in the uppermost reaches and gravel accumulation further downstream, the following river engineering works were put into effect:-

1. The longitudinal river profile was stabilised by subdivision into a number of sections at the equilibrium slope for the river bed material, each section being separated from the next by a permanent si'l.

2 The river banks, consisting of erodible alluvium, were stabilised by maintaining the river bend radii in either direction at least five times the river width, which almost invariably reduced river bank protection require. ments to their simplest expression, and frequently eliminated them altogether on the convex side of the bends.

Except for sills exceeding $1.5 \mathrm{~m}$ in height, which are based on Creager's design, i.e. usually with a paved energy-dissipation basin, most of the river control structures are built of rubble. This method was originally developed in the Austrian Alps about twenty years ago, with artificial ramps usualy with a slope of $20 \%$ and a width limited to $1 / 20$ th of that of the river. In plan, the control structures form a curve with a radius equivalent to 1 lit imes that of the river width, which is designed to keep the flood discharge in the centre of the river downstream of the control structure.

The ramps are fashioned from large lumps of granite of at least 1 ton weight, which are placed side-by-side in the form of a particularly rough "hedgehog" structure capable of efficiently absorbing the full kinetic energy of the water. The upstream row of boulders is encased in concrete, whilst the downstream row is maintained in position by one or two rows of rails rammed to a depth of $4 \mathrm{~m}$, into the river bed, spaced between 60 and $80 \mathrm{~cm}$ apart.

This system oflers the following advantages:-

- It does not mar the landscape;

- It is easily installed, as there are no foundations;

- No dewatering is required for work under water;

- It can undergo deformation without endangering the overall structure;

- Efficient aeration of the water assists self-purification;

- No interference with fish proceeding up-river.

The same material is used in smaller sizes (smaller by $100-300 \mathrm{~kg}$ ) for river bank protection.

The material was formerly placed by hand, proceeding "fish-scalewise" from downstream to upstream, but power shovels are now used for the purpose, except for placing the small stones inserted between the large blocks.

The foundations of the structure consist of rubble tipped into a pit with a trapezoidal cross-section.

To improve the appearance of the site, willow cuttings have been implanted (salix purpurea-usually in autumn) underneath the rubble bed, or more frequently standing more or less upright between the lumps of rock. Thus, after only a few years, the rubbie is now hidden behind a curtain of vegetation, which simply requires cutting back every five years. 